\title{
EDITORIAL
}

\section{MULTI DRUG RESISTANT TUBERCULOSIS}

Tuberculosis (TB) is the leading cause of death among people who are HIV-positive. Approximately one-third of the nearly 40 million people living with HIV / AIDS are also infected with TB. In areas such as sub-Saharan Africa, up to half of the AIDS-related deaths are caused by TB. During the early 1990s, multidrug-resistant tuberculosis (MDR TB), defined as resistance to at least isoniasid and rifampicin, the two most powerful anti-tuberculosis drugs emerged as a threat to TB control worldwide (1). The numerous outbreaks of MDR TB reported from many different countries located in diverse geographical environments during this time were indications of a global epidemic. Between 1994 and 2002, the WHO Global Project on Anti-TB Drug Resistance Surveillance coordinated data collection on more than 250,000 patients from 109 countries (or regions within large countries), representing $42 \%$ of the world's population (2). On this basis, WHO estimated the annual burden of MDR TB to be approximately 300,000-600,000 cases and the prevalence of MDR TB to be threefold higher than the annual incidence, primarily in low and middle-income countries. Mortality is significantly greater in HIV positive patients with MDR TB than those who are HIV negative (2-4). Very recently, the sudden emergency of XDR TB has become a significant cause for concern globally. XDR TB is defined as extensively drug resistant $\mathrm{TB}$ that is resistant to at least three of the six classes of the available second-line drugs, in addition to MDR TB (5).

In Kenya, findings from the 1995 anti-TB drug resistance surveillance showed that despite increasing prevalence of both TB and HIV-I infections, rates of initial drug resistance were still low and no MDR TB was reported (6). However, earlier results indicated that levels of drug resistance and MDR TB were higher in the refugee population than in the surrounding non refugee community in North-Eastern Province (7). Ten years later, MDR TB strains were first described in an indigenous patient population from private and public health care facilities in Nairobi $(8,9)$. MDR TB has also been observed in the last national anti-TB drug resistance surveillance conducted in 2002 (10), exactly 10 years since the introduction of Direct Observed Therapy Short course (DOTS) in the country. DOTS is a firstline drug regimen that includes isoniazid and rifampicin.

Drug resistance is largely a man-made phenomenon and is a consequence of suboptimal regimens and treatment interruptions. Other factors that commonly lead to development and transmission of MDR TB include HIV seropositivity, poverty and overcrowding, mixing of persons with TB not responding to therapy with HIV-infected persons in crowded hospital wards and clinics, failure to provide effective treatment support and assurance of adherence; failure to recognise and address patient non-adherence; adding a single new drug to a failing regimen and failure to recognise existing drug resistance $(2,11)$.

Patients with MDR TB are difficult to cure because they require prolonged hospitalisation and the use of second-line drugs that are toxic and more costly than first-line based regimens (2). The price of most second-line antituberculosis drugs recommended for use in the treatment of MDR TB is unaffordable to countries in need. In Kenya, it is currently estimated that over US $\$ 18,000$ is required to treat a single case of MDR TB (Sitienei, personal communication, 2006) compared to approximately US $\$ 400$ required to treat a non MDR TB case.

In 2000, the WHO-based Stop TB Partnership's Green Light Committee (GLC) was created to increase access to second-line drugs worldwide while ensuring their proper use to prevent the spread of increased drug resistance. During the time, this Scientific Panel of the WHO Working Group on DOTS-Plus for MDR TB produced its first set of guidelines - Guidelines for establishing DOTS-Plus pilot projects for the management of multidrug-resistant tuberculosis - which were reviewed in 2005 (2). The new guidelines provide guidance on current best practice in the management of drug-resistant TB, especially MDR TB, that should be adopted worldwide. It is emphasised that these recommendations should be followed without hesitation by all national TB control programmes and their partners as the most solid programmatic 
standards. At the same time, it is important to highlight that the new global TB control strategy remain the cornerstone of TB control and the most effective tool for preventing the onset and dissemination of drug resistance. The overall TB control strategy will consist of six key components. The first DOTS strategy's key elements will include political commitment, passive case finding, standardised chemotherapy, regular supply of drugs and monitoring and evaluation. The other key components of the control strategy include MDR TB/HIV and emerging challenges, involvement of all stakeholders - Public-Private Mix (PPM), strengthening of health care systems, involvement of communities and patients (patient charter) and operational research. Without these essential elements of TB control fully in place, management of MDR TB will undoubtedly fail in the long term (2).

While new strategies for containing MDR TB are now in place, the emergency of XDR TB is reported to have a wide geographic distribution and is associated with worse treatment outcomes than MDR TB (MMWR) (11). In Africa, virulent strains of TB resistant to all standard drugs have killed 52 of 53 patients in a rural hospital in South Africa in recent months (12). This super-resistant strain of TB has up to now been very rare, making an outbreak in 53 people catastrophic. While resistant TB often arises from incomplete or ineffective drug treatment, surprisingly, half of the patients with MDR TB in South Africa had never been treated for TB before, a perturbing sign that they were newly infected with the extreme strain and that XDR is swiftly being transmitted in the community. Furthermore, 44\% of the patients were HIV-positive, severely immunocompromised and resulted in $98 \%$ of them dying within one month of submitting an initial sputum sample. The median survival time was a mere 16 days. In the same outbreak, two healthcare workers also died of MDR TB. In addition to this, very recently, a report in a Kenyan local newspaper indicates that 263 cases with at least 30 new cases of XDR TB are being reported each month in KwaZuluNatal, where almost $40 \%$ of the adult population is HIV infected (13). The situation in other African countries is yet to be known.

In the only previously-published study on the XDR strain, the U.S. Centers for Disease Control (CDC) and Prevention earlier this year reported that from 2000 to 2004, the combined efforts of CDC and the WHO surveyed 17,690 collected samples of TB bacillus from the world's TB laboratories, and found a total of 353 cases or $2 \%$ of the collected patient samples were XDR (MMWR) (5). While TB is believed to kill about 2 million people worldwide each year, a growing number and proportion of XDR TB cases could seriously hamper TB control globally. Countries with good TB programmes have reported possible cure for XDR TB of only between $50 \%$ and $60 \%$.

In spite of the fact that the extent of drug resistance is still unknown in many countries and management of patients with MDR TB remains inadequate, in countries such as Kenya where drug MDR TB has been identified, specific measures need to be taken within TB control programmes to address the problem through appropriate management of patients and adoption of strategies to prevent the propagation and dissemination of drug-resistant TB, including both MDR TB and XDR TB. In addition to this, MDR TB and XDR TB can be prevented by ensuring that the national TB programmes and healthcare workers dealing with people with TB both in public and private sectors work in accordance with acceptable international standards for TB care. It is imperative to note that all the drugs used for treating TB have been in use for a long time and if they are not properly used, tubercle resistance to them will develop.

Therefore, appropriate use of these drugs for early treatment, in conjunction with adjunct measures such as early diagnosis and isolation of infectious cases must be ensured in order to contain the emergence of both MDR TB and XDR TB epidemics. Nevertheless, existing methods for drug susceptibility testing (DST) of second-line drugs for treatment of MDR TB are not only less reproducible than tests for susceptibility to first-line drugs but are also time consuming and this leads to further delays in obtaining results (14). Furthermore, WHO recommends that DST should be performed in specialised reference laboratories that participate in the current, rigorous quality assurance programme $(1,2)$. These facilities are not uniformly available in many resource-limited settings. In view of this, establishment and scaling up laboratory capacity is an immediate requisite. This approach will greatly improve detection and management of both drug susceptible and resistant TB (15). 
W.A. Githui, PhD, Principal Research Officer, Centre for Respiratory Diseases Research, Kenya Medical Research Institute, P.O. Box 54840, Nairobi, Kenya.

\section{REFERENCES}

1. World Health Organisation/International Union Against Tuberculosis and Lung Disease Global Project on Anti-Tuberculosis Drug Resistance Surveillance. Anti-tuberculosis drug resistance in the world: report no. 3. Geneva, Switzerland: WHO. 2004.

2. World Health Organisation. Guidelines for the programmatic management of drug-resistant tuberculosis. Geneva, Switzerland: WHO. 2006. (WHO/HTM/TB/2006.361).

3. Fischl M.A., Daikos G.L., Uttamchandani R.B., et al. Clinical presentation and outcome of patients with HIV infection and tuberculosis caused by multiple-drug-resistant bacilli. Ann. Intern. Med. 1992; 117: 184-190.

4. Drobniewski F. Is death inevitable with multidrug resistant TB plus HIV infection? Lancet. 1997; 349: 71-72.

5. Centers for Disease Control and Prevention (CDC) emergence of Mycobacterium Tuberculosis with extensive resistance to second-line drugs - Worldwide, 2000-2004. MMWR. 2006; 55(11): 301-305.

6. Githui W.A., Juma E.S., van-Gorkom J., Kibuga D. and Drobniewski F., Antituberculosis drug resistance surveillance in Kenya, 1995. Int. J. Tuberc. Lung Dis. 1998; 2: 499-505.

7. Githui W.A., Hawken M., Juma E.S., et al. Surveillance of drug resistant tuberculosis and molecular transmission of resistant strains in refugee and non refugee populations in North-Eastern Kenya. Int. J. Tuberc. Lung Dis. 2000; 2: 235-241.

8. Githui W.A., Meme H., Juma E., et al. Isolation of multidrug resistant tuberculosis strains in patients from private and public health care facilities in Nairobi, Kenya. Int. J. Tuberc. Lung Dis. 2004; 8: 837-841.

9. Githui W.A., Jordaan A.M., Juma E.S., et al. Identification of MDR TB Beijing/W and other Mycobacterium tuberculosis genotypes in Nairobi, Kenya. Int. J. Tuberc. Lung Dis. 2004; 8: 352-360.

10. Githui W.A., Meme H., Muthami L., et al. Anti-tuberculosis drug resistance surveillance in Kenya, 2002, the $37^{\text {th }}$ Union World Conference on Lung Health: 31 October to 4 November 2006. Int. J. Tuberc. Lung Dis. 2006; Abstract No. PS-62001-62003.

11. Teixeira L., Perkins M.D., Johnson J.L., et al. Infection and disease among household contacts of patients with multidrug-resistant tuberculosis. Int. J. Tuberc. Lung Dis. 2001; 5: 321-328.

12. Altman L.K. Doctors warn of powerful and resistant tuberculosis strain THE NEW YORK TIMES: August 18,2006

13. McGregor S. and Ferry T. Hospital struggles with deadly new TB strain. Nation Newspaper, December 4, 2006.

14. Heifets L.B. and Cangelosi G.A. Drug susceptibility testing of Mycobacterium tuberculosis: a neglected problem at the turn of the century. Int. J. Tuberc. Lung Dis. 1999; 3: 564-581.

15. Smart T. Scaling up laboratory capacity for the coming storm of XDR-TB, and why the HIV treating community must get involved now. Nam, http:/ www:aidsmap.com 2006. 